\title{
Pensando en la memoria de las artes, el diseño y la arquitectura en el Ecuador: el caso LIPADA - PUCE
}

Thinking of memory of Ecuadorian Architecture, Design, and Arts: the case LIPADA - PUCE

Giada Lusardi

ISSN (imp): 1390-4825

Fecha de recepción: 03/2017

ISSN (e): 2477-9199

\begin{abstract}
Resumen:
LIPADA - PUCE constituye un laboratorio/archivo que conserva, investiga y difunde materiales que aportan a la consolidación de la memoria de la arquitectura, el diseño y las artes del Ecuador del siglo XX. Se construye a través de archivos privados donados a la Pontificia Universidad Católica del Ecuador, específicamente para el proyecto.

El presente artículo aborda la naturaleza de LIPADA en relación a su definición como laboratorio y archivo y las modalidades con las cuales se inscribe en el actual debate sobre archivos, tomando en cuenta una serie de aspectos que caracterizan la investigación en los ámbitos de la arquitectura, el diseño y las artes. Finalmente, se revisarán algunos modelos referenciales para pensar en su configuración, en la medida que reflexionan sobre la documentación del "proyecto", las modalidades de catalogación, digitalización, y difusión, así como la relación entre archivo y universidad.
\end{abstract}

Palabras claves: Archivo, artes, arquitectura, diseño, memoria, patrimonio, proyecto, laboratorio.

\begin{abstract}
:
LIPADA is a laboratory/archive, which seeks dialogue, study, and materials dissemination that contribute to Ecuadorian memory establishment, of twentieth century Architecture, Design, and Arts. It is formed with private archives that have been donated to Pontificia Universidad Católica del Ecuador, for this project. This article concentrates in LIPADA's disposition in connection to its description both as a laboratory and an archive, and the ways in which it is recorded on archives debate, considering a series of aspects, and depicting research within Architecture, Design, and Arts. Finally, some referential examples to think about the project, documenting procedures, digitalization, and the relation between archive and university.
\end{abstract}

Keywords: Archive, arts, architecture, design, memory, heritage, project, laboratory.

\section{Autor:a}

Giada Lusardi (Parma - Italia, 1984). Profesora Auxiliar a tiempo completo de la Pontificia Universidad Católica del Ecuador (Quito), donde imparte Historia del Arte del siglo XX y XXI, Curaduría de exposiciones de arte y Crítica del Arte. Máster en Historia del Arte Medieval, Moderna y Contemporánea en la Facultad de Letras y Filosofía de la Universidad de Parma (2012), en 2008-2009 ha realizado un periodo de estudio en el departamento de Historia del Arte de la Facultad de Historia y Geografía de la Universidad de Barcelona. Además, es Licenciada en Bienes Artísticos, Teatrales, Cinematográficos y de los Nuevos Medios en la Facultad de Letras y Filosofía de la Universidad de Parma (2008). Desde 2005 se ocupa de la gestión de proyectos culturales y museológicos, didáctica de museos y laboratorios de técnicas artísticas. 


\section{ECUADOR Y LOS ARCHIVOS}

En el Ecuador, si un investigador pretende escribir sobre la obra de algún arquitecto, diseñador, artista, fotógrafo, galerista o crítico del arte del siglo XX, se enfrentará seguramente a grandes dificultades debido a la escasez bibliográfica, a su difícil recuperación en las bibliotecas públicas y a las problemáticas en la gestión de los archivos. ¿Cuál archivo visitar? En el Ecuador existen varios archivos históricos importantes a los cuales acceder: el Archivo Nacional, el Archivo del Ministerio de Relaciones Exteriores, el Archivo Biblioteca de la Asamblea Nacional, el Archivo General de la Universidad Central del Ecuador, el Archivo Histórico Metropolitano de Quito, los archivos del Ministerio de Cultura y Patrimonio, la Biblioteca Archivo Aurelio Espinosa Pólit, el Archivo del Academia Nacional de Historia del Ecuador, los archivos de la Casa de la Cultura Ecuatoriana, el Archivo Histórico del Guayas, el Archivo del Instituto Metropolitano de Patrimonio, entre otros.

El problema mayor de nuestro investigador sería encontrar fondos documentales que guarden específicamente la memoria de las artes, el diseño o la arquitectura, ámbitos de estudio que normalmente no se asocian a la palabra archivo, sino a la de museo. ¿Dónde se conserva y quién en el Ecuador reúne, ordena y difunde el patrimonio documental vinculado a las artes, el diseño y la arquitectura del Siglo XX? ¿Qué pasaría si quisiéramos construir relatos alternos a los oficiales, los que encarnan los museos públicos, por ejemplo? ¿Cuáles instituciones podrían ofrecer una experiencia de interpelación distinta y sin jerarquías? ¿Cómo estudiar los procesos creativos de los artistas, diseńadores, ilustradores, arquitectos etc. sin los insumos que describan la etapa previa a la concreción de la obra final?
En las palabras "archivo y "archivar" no solo existe un sentido de origen, de iniciación, de una autoridad ordenadora asociada a un poder político, sino la idea de un lugar físico que garantiza la participación y el acceso a su configuración e interpretación, lo que sugiere un vínculo entre archivo y democracia (Derrida, 1996). Este vínculo históricamente, se traduce en un sistema legislativo capaz de tutelar y organizar la salvaguarda del patrimonio documental de la Nación y garantizar dicha democracia. El nexo archivo-democracia también justifica la configuración en las últimas dos décadas de una serie de archivos especializados en los ámbitos de las artes, el diseño y la arquitectura que intentan multiplicar las voces y las posibles narraciones.

Uno de los ejemplos más antiguos de archivo especializado es el Archivo Blomberg, un archivo privado fundado en el ańo 2000 y que conserva más de 30.000 negativos y diapositivas, fotografías, cartas y diarios de viaje, artículos periodísticos, revistas, libros, caricaturas, mapas y 36 filmes documentales del explorador, escritor, fotógrafo y cineasta Rolf Blomberg (Suecia, 1912 - Quito, 1996), además de las pinturas y documentos de su esposa, la pintora abstracta Araceli Gilbert (Guayaquil, 1913 Quito, 1993). Dedicados al audiovisual, existen unos pocos casos de archivos, entre los cuales destacan la Cinemateca Nacional Ulises Estrella, fundada en 1981 como un departamento de la Casa de la Cultura Ecuatoriana y que conserva más de 4000 títulos ecuatorianos, y un fondo documental que supera los 10000 documentos entre guiones, fotografías, afiches, así como una biblioteca especializada con libros y catálogos de cine. También cabe mencionar a la AANME (Asociación Archivo Nuevos Medios Ecuador) de María Belén Moncayo, que nace como iniciativa independiente en 2003 para conservar la 
documentación de videoarte y cine experimental de 290 artistas nacionales y extranjeros que residen en el Ecuador.

En el ámbito público, existe el proyecto del Archivo Nacional de Fotografía del Instituto Nacional de Patrimonio del Ministerio de Cultura del Ecuador, que desde el año 2014 organiza un archivo fotográfico digital que comprende la fotografía desde sus inicios en el país en 1840, hasta la segunda mitad del siglo XX. Cabe mencionar además, dos proyectos inactivos: 1) el Centro Ecuatoriano de Arte Contemporáneo (CEAC) y 2) el Museo Archivo de Arquitectura del Ecuador (MAE). El CEAC, creado en Quito en 1995 con el objetivo de impulsar la producción, difusión e investigación del arte en el Ecuador, ha implementado una base de datos digital con informaciones sobre artistas ecuatorianos contemporáneos, pero actualmente no se encuentra en línea. Por su parte, en el campo de la arquitectura destaca el MAE, un proyecto concebido por el Colegio de Arquitectos de Pichincha en colaboración con el FONSA (Fondo de Salvamento del Patrimonio). Este nació en los ańos noventa con la voluntad de documentar, conservar y difundir la Arquitectura y el Urbanismo de Ecuador a través de su archivo y de sus programas educativos y culturales, lastimosamente no pudo sostenerse financieramente y dejó de funcionar.

En consecuencia y a partir del interés en la contribución al panorama de los archivos y específicamente de los archivos especializados en los ámbitos de la investigación de las Artes, el Diseño y la Arquitectura del Ecuador del Siglo XX, se configura en el año 2016, LIPADA, Laboratorio de Investigación sobre fondos documentales del Proyecto de Arquitectura, Diseño y Artes del Ecuador en el Siglo XX, en la Pontificia Universidad Católica del Ecuador.

\section{IDEAS SOBRE EL PROYECTO LI-}

\section{PADA}

LIPADA nace a raíz de una serie de investigaciones financiadas por la Pontificia Universidad Católica del Ecuador a partir del año $2007^{1}$, que evidenciaron la necesidad de crear un espacio físico en el cual conservar una serie de fondos documentales personales, vinculados a las artes, el diseño y la arquitectura en peligro de desaparición, debido a la ausencia en el país de un lugar especializado para su conservación, investigación y difusión.

La PUCE, desde un inicio, apareció como el lugar que podía acoger estos acervos dada su tradición de custodia de importantes colecciones de arte como las de Jacinto Jijón y Caamaño, José Gabriel Navarro y Carlos Bossano, las colecciones arqueológicas de los esposos Weilbauer, del padre Pedro Porras, la colección de Costanza di Capua los fondos documentales de José María Velasco Ibarra, el Archivo-Museo Juan José Flores y, en el ámbito de las ciencias, el Museo de Zoología QCAZ de Vertebrados.

Además, la PUCE desde el año 2013 costea otro proyecto de documentación y visibilización de la producción artística contemporánea ecuatoriana llamado INDEX: conversaciones de Arte Contemporáneo, un archivo audiovisual que nace por iniciativa de los profesores de la Carrera de Artes Visuales, Jaime Sánchez y José Luis Macas. Estos proyectos y colecciones me animaron junto a mi colega Shayarina Monard, a confiar en la Institución y proponer a LIPADA como un espacio para rescatar, conservar, investigar y difundir la producción creativa ecuato-

1 "Karl Kohn/ Arquitecto, diseñador, artista" (Monard, 2010, ISBN: 978-9978-77- 168-6); "Ovidio Wappenstein" (Monard, 2010, ISBN: 978-9978-8698); "Milton Barragán Dumet/ obras que perviven" (Monard, 2013, ISBN: 978-9942-21- 868-1); y el "Proyecto de creación del archivo documental de La galería de Beatriz Deller Maier" (Lusardi, 2015, en ejecución). 
riana en los ámbitos de la arquitectura, del diseño y de las artes del siglo XX.

LIPADA consiste al mismo tiempo en "laboratorio" y "archivo". "Laboratorio", porque se concibe en primer lugar como un espacio dinámico de experimentación y generación de nuevas aproximaciones teóricas sobre la producción creativa de arquitectos, diseñadores y artistas. Por otra parte es "archivo", en cuanto se constituye como un espacio que conserva, investiga y difunde las memorias custodiadas en sus documentos, mostrando la riqueza y complejidad de la historia. Este "archivo", se concibe también como una estructura que no posee un orden de lectura predefinido y se configura como un "sistema" abierto a múltiples recorridos e investigaciones. El archivo además, no prefigura un ordenamiento interno, y siquiera ofrece un modelo narrativo, sino que invita a ser interpelado de una forma personal, donde cada individuo puede elegir entre las muchas estructuras narrativas posibles o los varios modelos de búsqueda, aquellos que les permitan traer un sentido a los documentos, textos o imágenes, con los cuales se confronta.

LIPADA invita a investigar sin aplicar jerarquías y cuestionando los límites disciplinares con el fin de comprender la realidad, los contextos y los sistemas del arte que permitan ver, por ejemplo, en una fotografía, afiche o invitación a una exposición etc. no solamente su valor documental, sino su valor estético. También se trabaja a partir del concepto de "fondo documental", enfatizando un interés por mantener la integridad de los archivos privados una vez trasladados y, en la medida de lo posible, su ordenamiento original, con la idea de que en esta configuración original, se refleja el mundo de su autor y permite entender la concepción del mundo de sus creadores.
Para poder entender la naturaleza física de las colecciones conservadas en LIPADA, es necesario ofrecer algunos datos. Hasta ahora se cuentan con alrededor de 2000 planos arquitectónicos, 100 dibujos, 1000 unidades entre fotografías, negativos y slides, 3 maquetas, 229 carpetas con documentos de varias tipologías, 261 álbumes de documentación de exposiciones, 200 pruebas de imprenta, 5 libros de visitas a exposiciones, 2000 unidades entre catálogos, invitaciones, material de difusión para ferias de arte, postales, recortes de prensa, revistas, libros impresos, entre otros. LIPADA posee también los archivos de "la galería" de Beatriz Deller Maier (Quito, 1977 - 2001) y los archivos de los arquitectos Ovidio Wappenstein (Canals, España, 1938) y Karl Kohn (Praga, 1894 - Quito, 1979). Asimismo, prevé incorporar cada año un número limitado de fondos documentales, bajo criterios de selección basados en la relevancia del archivo privado y el rol del creador en sus respectivos campos de producción. Estos protocolos de adquisición junto con los otros necesarios para el correcto funcionamiento de cualquier archivo, contribuyen a la consolidación de este espacio de investigación como un espacio institucional al servicio de la comunidad.

\section{LOS MODELOS REFERENCIALES PARA LIPADA}

Durante la configuración de LIPADA, se generaron numerosos debates con otras instituciones acerca de 1) la idea de documentación del "proyecto", 2) la identificación de las modalidades de catalogación, 3) la digitalización y 4) la relación entre archivo y universidad. Estas discusiones configuraron una red de categorías válida para la definición de aspectos conceptuales y técnicos. Es importante, por lo tanto, especificar que los modelos referenciales que se presentarán 
a continuación han sido identificados durante el proceso de implementación del laboratorio/ archivo y responden a intereses estrictamente pragmáticos.

\subsection{Pensando en la documentación del "proyecto".}

Alrededor de 1968, Arturo Carlo Quintavalle, profesor de lo que en aquel entonces era el Instituto de Historia del Arte de la Universidad de Parma, plantea la necesidad de conservar la memoria, o sea, el documento del "proyecto", el tejido histórico del pasado. Esto lo llevó a pensar en algo que actualmente parece muy obvio: ¿cómo archivar la fotografía, lenguaje por naturaleza reproductible? De esta manera, Quintavalle se percató sobre la necesidad de recolectar las huellas de los procesos de investigación de los artistas, arquitectos, diseñadores y otros, estableciendo con el paso del tiempo el actual CSAC o Centro Studi e Archivio della Comunicazione de la Universidad de Parma, que conserva actualmente alrededor de 12.000 .000 de piezas.

Desde un inicio, LIPADA se configura a partir de este mismo interés por conservar la "documentación del proyecto", y enfatizar en la exploración del carácter procesual de la creación moderna y contemporánea que, a largo del siglo $\mathrm{XX}$, ha explorado repetidamente el territorio de la inmaterialidad haciendo énfasis en la cuestión del "proceso". Teniendo en cuenta la naturaleza efímera de muchos proyectos artísticos contemporáneos, existe la necesidad de un registro y una memoria que, a menudo, proviene de testimonios escritos y visuales, que representan las únicas huellas para articular una historiografía crítica del arte contemporáneo. El proyecto de arte contemporáneo necesita, por lo tanto, de su documentación para ser estudiado. Este cambio en la forma de concebir el arte es el que ha lle- vado en los últimos años a la configuración de numerosos centros de documentación asociados a museos de arte contemporáneo.

En São Paulo existe desde el año 2006, el Centro de Documentación del Instituto de Arte Contemporáneo (IAC) que preserva, cataloga y brinda acceso público a los archivos personales de los artistas brasileños modernos y contemporáneos pertenecientes a la colección. Es el caso de Sergio Camargo, Willys de Castro, Amilcar de Castro, Sérvulo Esmeraldo, Lothar Charoux y Luis Sacilotto. Los archivos del IAC tienen alrededor de 26,000 artículos centrados en torno a esos artistas y los fondos incluyen correspondencia con los artistas, familiares, amigos e instituciones; documentación sobre exposiciones y publicaciones; recortes de prensa y opiniones; así como, programas de seminarios y simposios. Su colección consta de otros elementos originales como certificados, premios y reconocimientos, contratos con galerías, poemas, recibos, fotografías, carteles, invitaciones, guiones de cine, estudios de obras, bocetos, notas, moldes de obras y las biblioteca personales de los mismos artistas.

A lo largo de nuestras investigaciones, enfocadas en la conformación de esta idea de conservar el proyecto, se encontraron otros espacios interesados en pensar, por ejemplo, en la memoria proyectual del Diseño. Este es precisamente uno de los intereses principales del -I-D-A en Buenos Aires, fundado en 2013 por Gustavo Quiroga y Raúl Naón. Es una plataforma dedicada a la preservación, clasificación y promoción de la historia y herencia del diseño Argentino, que conserva una colección heterogénea de archivos y objetos, entre los cuales destacan los archivos de algunas importantes agencias de diseño, documentos de la Feria de América (una feria industrial que se abrió en 
Mendoza en 1954), la colección del Instituto Torquato Di Tella, la del Instituto de Diseño Industrial y del Centro de Investigación en Diseño Industrial (CIDI), entre otros. El caso de la conservación de la memoria de diseño es particularmente interesante debido a la naturaleza reproducible de sus productos. El diseño se sustituye, se desecha y por lo general es muy difícil pensar en su estudio, pasadas unas décadas de la fecha de su producción. Esto justifica la importancia de conservar sus productos y de documentar los procesos creativos que llevaron el diseñador a la obra final.

Los materiales proyectuales también permiten pensar en la historia de una arquitectura no construida o perdida, o en proyectos de arte o diseño nunca expuestos. En esta perspectiva trabaja el museo digital MORE (Museum of refused and unrealised art projects), de Elisabetta Modena y Marco Scotti, que colecciona, conserva y exhibe proyectos de arte rechazados o no realizados del siglo XX y XXI. En esta línea es necesario mencionar el proyecto Agency Of Unrealized Projects (AUP) de e-flux y de la Serpentine Gallery de Londres, curado por Hans Ulrich Obrist, Julia Peyton-Jones, Julieta Aranda y Anton Vidokle, así como la idea de un Museo de los Proyectos ya planteada en 2004 por Marina Pugliese en la vigésima conferencia internacional del ICOFOM o Comité Internacional para la Museología.

\subsection{Pensando en las modalidades de catalo- gación, digitalización y difusión.}

Para la configuración de un archivo, se requiere dedicar tiempo al estudio de las plataformas para la catalogación y la digitalización del patrimonio, de los lenguajes de interpelación y contextualmente, a la definición de las herramientas de indexación. Por esta razón, LIPADA ha ini- ciado una revisión de los sistemas de catalogación utilizados por los archivos más importantes a nivel nacional, entre los cuales destacan los archivos del Ministerio de Cultura y Patrimonio, que conservan varias colecciones anteriormente de propiedad del Banco Central del Ecuador.

El Banco Central del Ecuador, responsable de los bienes hasta el 2010, año del traspaso al recién nacido Ministerio de Cultura y Patrimonio, catalogó sus bienes con un sistema informático llamado RBC (Registro de Bienes Culturales), mientras que el fondo fotográfico de la misma institución trabaja desde los años noventa con el sistema WINISIS de la UNESCO. El mismo sistema, ya obsoleto (funciona solamente en computadoras con sistema operativo Windows 7 de 32 bits), se sigue utilizando en la Biblioteca Municipal de Guayaquil, una de las más antiguas del país, que conserva la biblioteca personal de Pedro Carbo y de don Juan B. Destruge. Actualmente, las instituciones museísticas públicas del país, utilizan el sistema CBC (Catálogo Digital de Bienes Culturales) para la catalogación de sus colecciones.

Durante el estudio para la identificación de los campos definitivos de la ficha catalográfica de LIPADA, se revisaron los modelos aplicados en las colecciones de arte del MAAC (Museo Antropológico y de Arte Contemporáneo) de Guayaquil, que conserva la mayor colección de Arte Moderno y Contemporáneo del Ecuador, y del Centro APICE de la Universidad de los Estudios de Milán. El interés en el MAAC viene dado por la relevancia de su colección cuyas obras se catalogan con una ficha que se concibió años atrás, cuando la colección pertenecía al Banco Central del Ecuador. Esta ficha enfatiza los aspectos estético-visuales de las piezas en concordancia con las fichas de catalogación de los bienes histórico-artísticos conservados en los museos de todo el mundo. 
Por otra parte, el interés por APICE se debe a las especificidades de sus colecciones bibliográficas y archivísticas enfocadas en el estudio de la literatura, el arte y la editorial moderna y contemporánea que posee materiales muy similares a los de LIPADA. Este centro, inaugurado en el ańo 2002, prevé por un lado, la catalogación archivística en un sistema llamado XDAMS y, por otro, una catalogación bibliográfica más tradicional, dentro de la cual se ha realizado un gran esfuerzo por incluir una catalogación iconográfica ICONCLASS, que pone énfasis en el análisis de las imágenes.

En lo que respecta a LIPADA, se trabaja actualmente con una ficha catalográfica genérica para todos los materiales, concebida a partir de la aplicación de las normas internacionales de descripción archivística (ISAD) (G) y que retoma algunos campos del modelo del MAAC y otros de APICE, con el objetivo de describir adecuadamente los diversos materiales conservados. En LIPADA las fichas se conciben por niveles, desde la ficha macro de descripción del fondo, pasando por fichas de las unidades documentales compuestas, por ejemplo, por proyectos arquitectónicos, álbumes de fotos o carpetas, hasta llegar a lo micro, representado por las fichas de las unidades documentales simples, como fotografías, dibujos sueltos, invitaciones etc.

La actividad de un archivo está estrictamente conectada con la reflexión sobre la creación de recursos digitales que sobreviven principalmente en función de su visibilidad. ¿Cómo organizarlos? ¿Cómo conservarlos? ¿Como difundirlos?

LIPADA se encuentra actualmente implementando un repositorio en DSPACE, estructurado por "árboles" que tienen la función de traducir la disposición física de los documentos, respetando la complejidad de los fondos documentales originales. En un futuro próximo, el laboratorio-archivo incorporará en el mismo repositorio digital la vasta documentación digital de la Arquitectura Ecuatoriana del Siglo XX, recopilada en la última década por la historiadora de la PUCE Shayarina Monard, investigadora también de LIPADA. Esta decisión responde a la imposibilidad de incorporar físicamente todos los archivos personales relevantes de nuestros campos de estudio y a la voluntad de salvaguardar ese patrimonio mediante un repositorio digital lo más completo posible.

Para pensar en la digitalización de los materiales y en el repositorio digital, LIPADA ha estudiado algunos modelos como el de la Benson Collection que, en los últimos años, ha intentado conseguir que materiales raros y de formatos especiales, estén fácilmente disponibles para estudiarse a través de iniciativas digitales. La Benson Collection lo logra supervisando la gerencia de registros y archivos en colecciones externas, para después digitalizar sus colecciones y crear un archivo digital único.

En el ámbito digital, otro proyecto referencial para LIPADA ha sido el Documents of 20th-Century Latin American and Latino Art. A Digital Archive and Publications Project, liderado por el International Center for the Arts of the Americas del Museum of Fine Art de Houston, que desde mediados de la primera década del siglo XXI se dedica a ubicar, clasificar y digitalizar documentos claves de la historia del arte Latinoamericano del siglo XX. Inspirado en este archivo, LIPADA ha implementado un repositorio que permite la descarga en formato PDF de los materiales.

En relación a la accesibilidad de la información, uno de los archivos que interesa particularmente a LIPADA es el CCP, The Center for Creative Photography de la Universidad de Arizona, una de las instituciones más trascendentes en el ámbito de la fotografía. El CCP es 
al mismo tiempo un centro de investigación de la historia de la fotografía, un museo de arte académico, el depósito de archivos de la más grande colección de fotografía norteamericana del siglo XX y un museo electrónico con más de 100,000 imágenes digitalizadas. Las obras del museo electrónico están organizadas por categorías, lo cual le permite al público explorar la colección con mayor libertad, y no solamente buscando fotografías por autor.

\subsection{Pensando en la relación archivo y univer- sidad.}

Un aspecto que desde el inicio ha sido fundamental para LIPADA, es la necesidad de pensar el archivo en relación con la academia, entendiendo las colecciones universitarias como lugares de encuentros, intercambios y formación de jóvenes. La presencia de colecciones en las Universidades invita a que se dé un diálogo constante con los estudiantes y ofrece posibilidades de investigación y formación de nuevas generaciones capaces de comprender y participar en el proyecto.

El archivo permite pensar en nuevas herramientas y metodologías para construir relatos y potenciar las sinergias entre profesionales de distintos campos, como historiadores - biólogos, para el estudio de las patologías de deterioro de los documentos; restauradores - economistas de la cultura, para pensar soluciones de sustentabilidad del proyecto; archivólogos para potenciar las posibilidades de comunicación del archivo, entre otros. En este sentido han sido importantes para LIPADA, el CSAC de la Universidad de Parma, APICE de la Universidad de Milán y el centro de documentación Arkheia, que está asociado al Museo Universitario de Arte Contemporáneo (MUAC) en la Universidad Nacional Autónoma de México. Arkheia actúa como un laboratorio de discusión e intercambio entre estudiantes, artistas, curadores e investigadores, y genera narrativas espaciales que invitan al espectador a considerar de modo diferente los materiales de los archivos y las jerarquías tradicionales entre documentos y objetos de arte.

Otro proyecto que ha sido referente para entender las posibilidades de los vínculos entre colecciones y academia es ESCALA (Essex Collection of Art from Latin America) ${ }^{2}$, que pertenece a la Universidad de Essex en Colchester (Reino Unido), y que a lo largo de los años ha generado un vínculo estrecho entre sus colecciones, sus programas de postgrado y el panorama de la investigación académica. ESCALA solicita, por ejemplo, a los estudiantes de uno de sus programas de postgrado que emprendan la investigación de una adquisición potencial, para someter esa propuesta a sus compańeros y a un panel de expertos o, invita a estudiantes, investigadores y académicos de toda la Universidad a seleccionar, reflexionar y escribir sobre piezas de la colección que están ligadas a sus propios intereses académicos.

LIPADA retoma este interés por potenciar las posibilidades de interacción del laboratorio-archivo con los usuarios a través de la utilización de sus materiales para construir nuevas experiencias en el aula que favorezcan el aprendizaje.

\section{CONCLUSIONES}

Las experiencias de los archivos presentados en este estudio, evidencian la necesidad de potenciar redes nacionales e internacionales para cuestionar constantemente las aproximaciones críticas al debate sobre la memoria de las ar-

2 ESCALA conserva un total de 750 obras, y una amplia gama de archivos que incluyen catálogos, correspondencia, currículums de artistas, material audiovisual, grabaciones y fotografías. 
tes, el diseño y la arquitectura, y para pensar en nuevos formatos para el archivo, abiertos al uso multifacético de sus colecciones. Estos espacios se caracterizan por concebir el archivo no solo como lugar de la memoria, sino como territorio de interacción social y de reflexión que, prescindiendo de las jerarquías, pone énfasis en los procesos creativos comunes a las artes y en el rol del individuo, coleccionista o creador como sujeto de investigación.

En concordancia con lo dicho, LIPADA reconoce la importancia de la conservación de la materialidad del documento como portador de una fuerte carga afectiva, estética e histórica. Por esto apoya la investigación en los campos de las Artes, el Diseño y la Arquitectura y ofrece un "hogar» a valiosos acervos documentales que, por su data o invisibilidad académica, aún no poseen el carácter de "históricos" y que por lo mismo corren el riesgo de ser desmembrados y desaparecer.

LIPADA justifica su existencia en estricta relación con la Academia, espacio en el cual se inserta y con el cual dialoga diariamente para activar nuevas formas de configuración de conocimientos, investigación y difusión en los campos de las artes, el diseño y la arquitectura.

Finalmente, LIPADA evidencia la necesidad de activar un diálogo a nivel nacional sobre la validez del archivo especializado como plataforma para la protección del patrimonio, como espacio educativo y de apoyo a la investigación y a la difusión de las artes, el diseño y la arquitectura ecuatorianos del siglo XX.

\section{Bibliografía.}

Archivo AANME - Archivo Nuevos Medios Ecuador. (s.f.). Recuperado el 14 de marzo de 2016, de http://archivo.aanmecuador.com/.

Archivo Blomberg. (s.f.). Recuperado el 14 de marzo de 2016, de http://www.archivoblomberg.org/.

Archivo Nacional de Chile. (s.f.). Recuperado el 14 de marzo de 2016, de http://www.archivonacional.cl/616/w3-channel.html.

Barriendos, J. (2009). (Bio)políticas de archivo. Archivando y desarchivando los sesenta desde el museo de arte. Artecontexto. (24). Recuperado de http://www.artecontexto.com/es/ver revista-24.html.

Braverman, L. (2016). Adquiriendo, Archivando y Activando: Un vistazo a la forma en la que diversas colecciones de arte latinoamericano son activadas en catorce instituciones distintas. Colección Cisneros. Recuperado el 30 de marzo de 2017, de http://www.coleccioncisneros.org/es/ editorial/cite-site-sights/adquiriendo-archivando-y-activando-parte-2.

Carnevale, G. (2009). Algunas interrogaciones sobre el archivo. En Freire, C., Longoni, A. (orgs.). Conceptualismos del Sur/Sul. (pp. 25 - 51). São Paulo, Brasil: Annablume y USP-MAC, AECID.

Carpenter Center for the Visual Arts. (s.f.). Harvard University. Recuperado el 14 de marzo de 2016, de http://ccva.fas. harvard.edu/.

Casa de la Cultura Ecuatoriana. (s.f.). Recuperado el 14 de marzo de 2016, de http:// www.casadelacultura.gob.ecl.

Centro APICE. . (s.f.). Universitá degli Studi di Milano.Recuperado el 14 de marzo de 
2016, de http://www.apice.unimi.it/.

Colegio de Arquitectos del Ecuador. Provincial de Pichincha. (s.f.). Recuperado el 14 de marzo de 2016, de http://www.cae.org. ecl.

CSAC. Centro Studi e Archivio della Comunicazione. (s.f.). Recuperado el 14 de marzo de 2016, de http://www.csacparma. it/.

Documents of 20th-Century Latin American and Latino Art. A Digital Archive and Publications Project. (s.f.). International Center for the Arts of Americas at the Museum of fine Arts, Houston. Recuperado el 14 de marzo de 2016, de http://icaadocs.mfah.org/icaadocs/.

Derrida, J. (1996). Mal d'archivio. Un'impressione freudiana. Napoli: Filema.

Ernst, W. (2016). Radically De-Historicising the Archive. Decolonising Archival Memory from the Supremacy of Historical Discourse. L'Internationale. Recuperado el 14 de marzo de 2016, de http:// www.internationaleonline.org/research/ decolonising practices $/ 50$ radically de historicising the archive decolonising archival memory from the supremacy of historical discourse.

Freire, C. (2009). Artistas/curadores/archivistas: políticas de archivo y la construcción de las memorias del arte contemporáneo. En Freire, C., Longoni, A. (orgs.). Conceptualismos del Sur/Sul. (pp. 64-82). São Paulo, Brasil: Annablume y USPMAC, AECID.

Furlus, E., Giannetti, C. (eds.). (2014). AnArchive(s). A Minimal Encyclopedia on Archaeology of the Arts and Media. Oldemburgo: Edith-Russ-Haus für Medienkunst.
Gómez, M. C. Archivo universal y derechos humanos: un estudio visual sobre la dialéctica de la mirada. (s.f.). Recuperado el 14 de marzo de 2016, de http:// www.flacso.org.ec/docs/i41gomez.pdf .

International Center for the Arts of the Americas, Museum of Fine Art of Houston. (s.f.). Recuperado el 14 de marzo de 2016, de www.mfah.org/icaa.

MAE (Museo Archivo de Arquitectura del Ecuador). (s.f.). Recuperado el 14 de marzo de 2016, de http://www.cae.org. ec/capacitacion/16-mae/26-museo-archivo-de-arquitectura-del-ecuador-mae. html.

Ministerio de Cultura y Patrimonio del Ecuador. (s.f.). Recuperado el 14 de marzo de 2016, de http://www.culturaypatrimonio.gob.ecl.

Monard, S. (2010). Karl Kohn/Arquitecto, diseñador y artista. Quito, Ecuador: Pontificia Universidad Católica (DVD).

Monard, S. (2013). Milton Barragán Dumet/ obras que perviven. Quito, Ecuador: Pontificia Universidad Católica. (DVD).

Monard, S. (1997). Ovidio Wappenstein. Quito, Ecuador: Pontificia Universidad Católica. (DVD).

Oña, L. (1997). La galería. Veinte años. Quito, Ecuador: Imprenta Mariscal.

Quintavalle, Arturo C., Bianchino, G. (2010). Nove100: arte, fotografia, architettura, moda, design. Milano: Skira.

Prelinger, R. (2009). The Appearance of Archives. En Snickars, P., Vonderau, P. (eds.), The YouTube Reader. (pp. 26874). Estocolmo: National Library of Sweden.

Red Conceptualismos del Sur. Estado de alerta. 
Los archivos en América Latina. (2009).

Instituto Europeo para Politicas Europeas

Progresivas. Recuperado el 14 de marzo

de 2016, de http://redcsur.net/tag/esta-

do-de-alerta/.

Rolnik, S. (2010). Furor de Archivo. ERRATA.

(1) Bogotá: FGAA, SCRD y Alcaldía Mayor de Bogotá.

Catela, S., Jelin, E. (2002). Los archivos de la represión: documentos, memoria y verdad. Madrid: Siglo XXI.

Barriendos, J., Gómez -Moya, C., Gutierrez - Castaneda, D., López, M., Videl, J. (2012). Micropolitics of the Archive Southern Conceptualisms Network and the political possibilities of microhistories. Archive as Method (02). Recuperado el 14 de marzo de 2016, de http:// www.aaa.org.hk/FieldNotes/Details/1208?lang=eng.

Yale University Art Gallery. (s.f.). Recuperado el 14 de marzo de 2016, de http://artgallery.yale.edu/.

Zanella, Francesa (2014). Digital archives. Alcune Note. Ricerche di S/Confine. (Dossier 3). Recuperado el 30 de marzo de 2016, de http:/www.ricerchedisconfine.info/ dossier-3/ZANELLA.htm. 